\title{
Modelos de poluição difusa: desafios, estratégias e impacto para a gestão de recursos hídricos
}

Submissão 06/07/2018

Revisão: $10 / 11 / 2018$ Aceite: 20/12/2018

\section{David Bispo Ferreira \\ Ana Paula Mühlenhoff \\ Cristóvão Vicente Scapulatempo Fernandes}

\section{RESUMO}

O controle de poluição apresenta-se como um desafio ao mesmo tempo técnico socioeconômico. A qualidade da água dos rios e reservatórios é reflexo direto das atividades desenvolvidas na bacia hidrográfica. O crescente grau de impermeabilização das bacias hidrográficas urbanas e o uso extensivo de insumos industriais, defensivos agrícolas e fertilizantes, além de produtos e serviços e atividades inerentes ao desenvolvimento são os principais fatores associados as fontes de poluição difusa nas bacias com características urbanas e rurais. Com origem no ciclo hidrológico a poluição por cargas difusas é bastante diversificada. Nesse contexto a modelagem de poluição difusa se apresenta como importante ferramenta de apoio ao processo de identificação das áreas geradoras desta poluição e ao processo de tomada de decisáo conjunto com a população e ao estudo de alternativas para a mitigação dos efeitos dos processos de ocupação na bacia e implementação dos instrumentos de gestão de Recursos Hídricos. O grande diferencial e desafio dos modelos de poluição difusa está na capacidade de representar as características de uso e ocupação do solo associadas àrepresentaçáo dos processos hidrológicos na bacia. A escolha do melhor modelo para um determinado problema deve considerar critérios bem estabelecidos. O presente trabalho apresenta uma revisão dos modelos de poluição difusa operacionais mais adequados a aplicação no contexto brasileiro, bem como constrói argumentos e critérios para a escolha de um modelo de poluição difusa, visando a implementaçáo dos instrumentos de Gestáo de Recursos Hídricos dentro da realidade brasileira. Adicionalmente, a partir da contextualizaçáo do problema de controle da poluição, apresenta os principais desafios e futuras direçōesem monitoramento e modelagem dos impactos da poluição difusa.

Palavras-chave:Modelagem de poluição difusa; gestão de recursos hídricos, Sistemas de informação geográficas

\section{ABSTRACT}

Nonpoint pollution is simultaneously a technical and socioeconomical challenge. Surface water quality is a direct consequence of activities developed within a watershed. The Increasing watershed impermeabilization and extensive usage of industrial chemicals, fertilizers and pesticides, in addition to products and services inherent to economic development are the main factors associated to nonpoint pollution inputs. As it takes place within the hydrological cycle, nonpoint pollution is very diversified regarding sources and pathways. Within that context, nonpoint pollution modelling is an important support tool to source area identification and to the decision-making process, aiming to achieve impact mitigation and implementation of water resource management instruments. A big challenge concerning the choice of a nonpoint pollution model must consider well defined criteria. This work presents a review of nonpoint pollution models, focusing on the implementation of water resource management instruments to the Brazilian reality. Additionally, it presents the main challenges and current directions of nonpoint monitoring and modelling

Keywords: Diffuse pollution modeling; water resources management, Geographic Information Systems 


\section{INTRODUÇÁO}

A poluição provém de aspectos sociais, como cultura, organização social e estilo de vida, que fomentam atividades relacionadas à economia, tais como agricultura, transporte e serviços. O meio ambiente provém recursos à sociedade e recebe impactos inerentes à utilização destes insumos naturais. Países em desenvolvimento são mais susceptíveis aos efeitos da poluição difusa, sendo agravados em centros urbanos pela ineficiência ou inexistência de sistemas de tratamento de esgoto e drenagem, associados a movimentos migratórios crescentes em direção a centros urbanos nem sempre acompanhados de medidas de saneamento apropriadas (NOVOTNY, 2003).

A criação e emprego de defensivos agrícolas e fertilizantes, além de outros complexos químicos utilizados em aplicaçóes industriais causa alteraçóes na especiação química dos mais diversos compartimentos ambientais (NOVOTNY, 2003), (NOVOTNY, 1999). Além disso, subprodutos de atividades como transporte, jardinagem e construção, contribuem significativamente com cargas de poluentes difusos (UNITED STATES ENVIRONMENTAL PROTECTION AGENCY, 1999; SHAVER et al., 1994). Atualmente, 58,2\% da população brasileira possui acesso a rede coletora de esgotos, sendo $68,2 \%$ deste total tratado (BRASIL, 2017). De acordo com os dados mais recentes do IBGE (INSTITUTO BRASILEIRO DE GEOGRAFIA E ESTATÍSTICA, 2008), até o ano da pesquisa, 76,8\% dos municípios possuíam rede de coleta de drenagem. Destes, $75,7 \%$ utilizam os corpos receptores como ponto final da rede. Destes municípios, $73,4 \%$ não possuem nenhum instrumento regulador do sistema de drenagem, sendo a regulamentação de uso e ocupaçáo de solo o instrumento de utilização mais freqüente.

A poluição difusa é definida como oriunda de atividades de uso do solo (urbanas e rurais), dispersas ao longo de uma bacia ou Sub-Bacia, e não são oriundas de efluentes industriais, de esgoto, mineraçáo e descarga pontual por atividades agrícolas (FERRIER, 2005; GOONETILLEKE et al., 2005). A qualidade dos corpos hídricos é influenciada por vários fatores, incluindo práticas de uso do solo, despejo de lixo e saneamento. Os mecanismos pelos cujos quais poluentes difusos são mobilizados no meio ambiente são importantes no desenvolvimento de estratégias de prevenção e controle dos mesmos (LEE; BANG, 2000). Uma quantidade significante de poluentes é gerada em bacias urbanas, desde poluentes grosseiros até toxinas solúveis são geradas em bacias urbanas (PARKINSON; MARK, 2005).

Os poluentes presentes no escoamento superficial variam entre bacias. Contudo, são associados a atividades específicas (e.g.: plantaçóes, atividades agropecuárias, construção civil) e usos do solo (e.g.: estacionamentos e áreas de serviços de automóveis). A maioria dos metais presentes no escoamento superficial urbano é provinda de ruas. Atividades agrícolas contribuem com uma gama de poluentes difusos, dependendo do tipo de produção e região. Em sua maioria são solos erodidos, fertilizantes, pesticidas, hidrocarbonetos e sais em solos (BURTON JUNIOR; PITT, 2002).

O ciclo hidrológico, assim como os seus diversos processos influenciam na quantidade e nos processos de transporte e destinação final de constituintes presentes no escoamento superficial. A água em movimento pode dissolver e mobilizar contaminantes no seu percurso. Os poluentes difusos são comumente separados em fase dissolvida, movendo por fluxos advectivos com a água, e uma fase aderida a sólidos (adsorvida). Além disso, processos de volatilização e particionamento, bem como difusão são importantes, tanto quando em movimento nas águas subterrâneas quanto superficiais (ESLAMIAN, 2014).

A identificação de fontes difusas consiste quantificação de descargas de poluentes e compreensão da sua destinação no meio ambiente. Embora nem todo problema de poluição necessite de modelagem e/ou simulação, são ferramentas úteis e amplamente utilizadas para o manejo sustentável na escala da bacia.

Modelos de poluição difusa não somente descrevem de maneira quantitativa, mas também podem permitiravaliar a distribuição temporal e espacial dos poluentes, sob várias fases e compartimentos ambientais (YANG; WANG, 2010). Dados específicos dos locais de estudo são necessários para preparação da análise de riscos, como concentraçóes oriundas de cada local, sua variação em relação às vazóes são necessárias para determinar as concentrações críticas em cada ambiente (BURTON; PITT, 2001).

Além disso, são freqüentemente empregados na avaliação da performance de medidas mitigadoras 
de volume e qualidade (MARTIN-MIKLE et al., 2015; KAINI; ARTITA; NICKLOW, 2007; GITAU; GBUREK, 2008; DAGGUPATI et al., 2010; ALMENDINGER; ULRICH, 2017), sejam elas náo estruturais (e.g..: regulamentação do uso do solo) ou estruturais (e.g.: construção de trincheiras vegetadas).A pesquisa com relação ao tema ainda é incipiente no Brasil, assim como em vários países do mundo (LI et al., 2014). Pesquisas conduzidas no Brasil (KOZAK; FERNANDES, 2016; LAABS et al., 2002; MAILLARD; PINHEIRO SANTOS, 2008; DOBLINSKI et al., 2010; APRÍGIO; BRANDÃO, 2013; RIGHETTO; GOMES; FREITAS, 2017) sugerem que existem corpos hídricos afetados por cargas difusas, o que pode indicar a necessidade da incorporação dos conceitos do manejo da qualidade e quantidade das águas precipitadas.

Este trabalho apresenta uma revisão dos principais desafios e tendências em relaçáo ao gerenciamento de cargas difusas em bacias, bem como uma apresentação e revisão de alguns dos principais modelos de simulação operacionais compatíveis com diferentes cenários típicos da realidade brasileira, notadamente ambientes com pouco ou nenhum monitoramento, e ambientes áridos no qual o balanço hídrico é complexo.

\section{DESAFIOS NA IDENTIFICAÇÃO, MONITORAMENTO E MODELAGEM DOS IMPACTOS DA POLUIÇÃO DIFUSA}

O processo de modelagem da poluição difusa está associado a uma série de desafios, uma vez que as fontes e os caminhos sáo de difícil rastreio. Alguns dos mais notórios e compatíveis com a realidade brasileira são: (1) Aquisição e disponibilidade de dados, (2) precisão de bases de dados e suas implicaçóes em parâmetros de modelagem, (3) capacidade de um modelo em reproduzir adequadamente usos do solo tipicamente urbanos, rurais e com ambos (4) performance do modelo em simular adequadamente regimes áridos (5) performance do modelo em simular com baixa quantidade de dados, conforme indicado na Figura 1.

Programas de monitoramento de poluição difusa são de elevado grau de complexidade. Trata-se de tentar representar um sistema através de parâmetros. Ao longo da condução do programa, estes resumem variadas interferências antrópicas de frequência e distribuição espacial desconhecidas. Os parâmetros monitorados necessitam de generalizaçóes para abranger maiores áreas da bacia, ou extrapolação visando avaliar efeitos temporais (WARDROPPER; GILLON; RISSMAN, 2017).

Outro ponto importante diz respeito à freqüência de coleta de dados, que deve ser determinada antes da aquisição. Esta frequência deve ser suficiente para que se possa atingir parcimoniosamente os objetivos de avaliação de impactos e medidas mitigatórias de maneira eficiente, a fim de que se obtenha compatibilidade entre os dados obtidos, e sua representatividade dentro de um estudo. Consequentemente, a captura da variabilidade dos processos durante o processo de monitoramento é uma tarefa complexa, custosa e que representa uma grande fonte de incerteza (FARZIN; KAPLAN, 2004). Contudo, nem sempre é possível estabelecer redes de monitoramento de parâmetros hidrológicos e qualidade da água de modo a se obter dados abundantes e de máxima adequabilidade aos modelos. Frequentemente, dados hidrológicos de outras estaçôes devem ser importados para aprimoramento

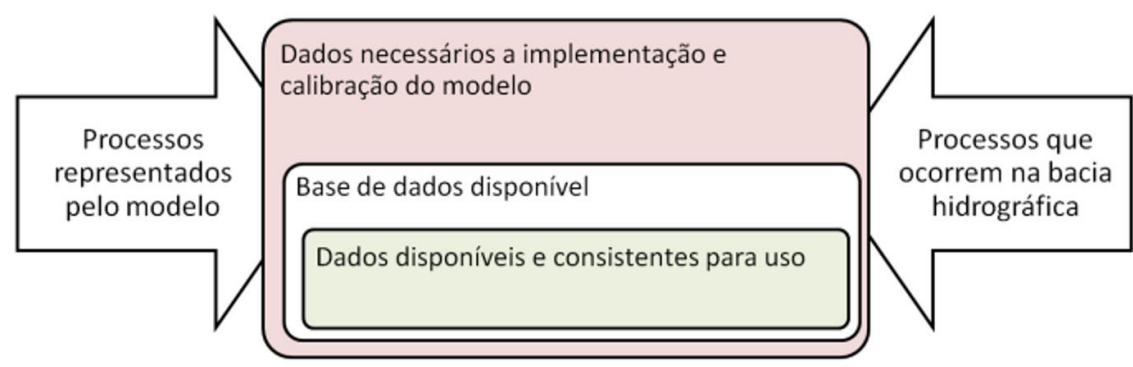

Figura 1 - Desafios associados a implementação, calibração e simulação de modelos de poluição difusa 
da modelagem hidrológica (WANG et al., 2011), o que também é uma grande fonte de incerteza.

Vários estudos têm sido conduzidos no que tange a aplicação de modelos de poluição difusa em locais sem ou com pouco monitoramento (ROMAGNOLI et al., 2017; DIHALA et al., 2012; OZCAN et al., 2017b; PANAGOPOULOS et al., 2011). Estes estudos majoritariamente baseiam-se em valores de literatura, sendo muitas vezes subsidiados por bases de dados locais, consistindo em modelos digitais de terreno (MDT), mapas de uso do solo, e técnicas indiretas de medição de parâmetros, utilizando técnicas como sensoriamento remoto, imagens hiperespectrais (LIN et al., 2015) e Lidar (WILLIS et al., 2016).

Bases de dados geoespaciais públicas são frequentemente empregadas, e fornecidas por entidades nacionais e internacionais, e permitem modelagem em áreas com baixa disponibilidade e/ou falta de monitoramento. Özcan et al. (2017a) demonstrou utilizando o Soil and Water Assessment Tool (SWAT) utilizando valores de referência na literatura para uma Bacia na Turquia que é possível obter resultados razoáveis de simulação hidrológica, e obter valores úteis de qualidade da água para sedimento, e nutrientes. Ora, se por um lado é possível e não menos importante compreender sistemas ambientais com baixa disponibilidade, por outro, dados resoluçôes maiores podem não fornecer necessariamente melhores resultados em termos de simulação hidrológica e de qualidade da água (SHEN et al., 2013; TAN et al., 2015; YANG et al., 2014).

Apesar de o monitoramento fornecer dados de entrada mais consistentes, a performance dos modelos de poluição difusa utilizando poucos dados fornece resultados poderosos no que tange a esforços de identificação e quantificação de processos de transporte de runoffe de seus constituintes. Harnmel et al. (2014) divide o emprego destes modelos em três categorias: exploratório, planejamento e regulatório/legal. Cada uma destas etapas necessita de diferentes quantidades e frequências de dados para assegurar uma representação verossímil do sistema.

Existem técnicas específicas para identificação e do potencial de exportação de poluição difusa, e estudo da viabilidade da mitigação, nomeadamente (1) Métodos de coeficiente de exportação, (2) Modelos de índices e (3) modelos de simulação de bacias. Modelos de coeficientes de exportação têm sido usados com sucesso (OMERNIK, 1976; BORAH; BERA, 2003) para quantificar impactos de usos do solo na qualidade das águas superficiais. Os modelos de índices levam em conta aspectos sociais, análises custo benefício, entre outros, sendo muitos deles baseados em técnicas multicritérios. Em outras palavras, visam classificar as áreas de risco e auxiliar o processo de decisão (HAJKOWICZ; COLLINS, 2007).

Um exemplo é o PLOAD (UNITED STATES ENVIRONMENTAL PROTECTION AGENCY, 1993), que calcula cargas anuais de poluentes difusos e tem sido empregado em vários estudos (SHI et al., 2012; GURUNG; GITHINJI; ANKUMAH, 2012; SHEN et al., 2011; LIN et al., 2016). O Potential Non Point Pollution Index (PNPI) (MUNAFÒ et al., 2005) utiliza indicadores de uso do solo, indicador de runoff, e de distância, computados através de uma fórmula multicritérios para destacar áreas de potencial emissão de poluição difusa.

O Sensitive Catchment Integrated Modelling Analysis Platform (SCIMAP) é um software desenvolvido no Reino Unido, que trabalha com a acumulação do risco ao longo de uma rede hidraulicamente conectada em um ambiente de geoprocessamento, tendo os valores de risco arbitrados por experts (MILLEDGE et al., 2012).

O Agricultural Potential Pollution Index (APPI) (PETERSEN et al., 1991), efetua o ranqueamento de áreas agrícolas quanto ao potencial de emissão depoluição difusa utilizando quatro índices: um índice de escamento superficial, índice de produção de sedimentos, índice de uso de químicos e de população animal. Outros métodos como o de Koo e O'Connell (2006), propóem análises econômico-sociais para tomada de decisão.

Outro tipo de algoritmo são os modelos de simulação de bacias. Segundo Lane et al. (2006), permite-se fazer outra classificar modelos de simulação poluição difusa em: (1) Funçôes de inferência, ou seja, modelos nos quais se busca correlaçóes entre dados de exportação e observaçôes de campo, obedecendo a leis de balanço de massa, (2) Funçóes de transferência, na qual se obtém a exportação de poluentes através 
de taxas de exportação e modelos de exportação de massa (3) Modelagem de da unidade de solo: aplicam leis físicas ou conceituais para modelagem da transferência de poluentes e (4) Modelagem de transferência de solo: combina a abordagem física de (3) com algoritmos dinâmicos para descrição dos aportes de cargas(i.e.:rotação de culturas, aplicaçóes periódicas de pesticidas e fertilizantes).

Dentro desta realidade, a maioria dos modelos de simulação de bacias são baseados em modelos hidrológicos acoplados a modelos de transporte e destinação de poluentes. Estes modelos obedecem a equaçôes de balanço hídrico, balanços de massa, acoplados a relaçóes constitutivas de contorno e funçóes que descrevem comportamentos específicos (i.e.:equações de perda de solo, exportação e transformaçáo de nutrientes e pesticidas). A Figura 2, a seguir demonstra conceitualmente o funcionamento dos modelos de simulação

Modelos de poluição difusa são frequentemente vinculados a dados de Sistemas de Informaçôes Geográficas (SIG). Muitos modelos, tanto de índice (PNPI, SCIMAP), quando de simulação de bacias (SWAT,
HSP-F, SWMM, AGNPS, AnnAGNPS, STORM) são compatíveis com interfaces de Sistemas de Informação Geográfica (SIG), facilitando o processo de entrada de dados (BORAH; BERA, 2003). As interfaces SIG ocupam em diferentes algoritmos, podem ocupar papéis (1) secundários, sendo seus recursos de levantamento de informaçóes geoespaciais como ferramentas de preparação de entrada ou (2) primários, tendo seus recursos como insumo à modelagem da poluição difusa. Um exemplo é a utilização álgebra de grelhas vetorizadas ou algoritmos de otimizaçáo montados sobre bancos SIG (LIU et al., 2014).

A representação da estrutura geográfica de uma bacia hidrográfica é função da precisão requerida e da complexidade de sua estrutura topográfica e dinâmicas de uso do solo (ERTURK et al., 2007). Sendo assim, o equilíbrio entre o grau de detalhamento, o objetivo do programa de mitigação e a técnica empregada devem ser levados em consideração no planejamento do programa de mitigação de impactos de poluição difusa.

No Brasil, existem regióes de clima semiárido Estas regióes, representam um triplo desafio no

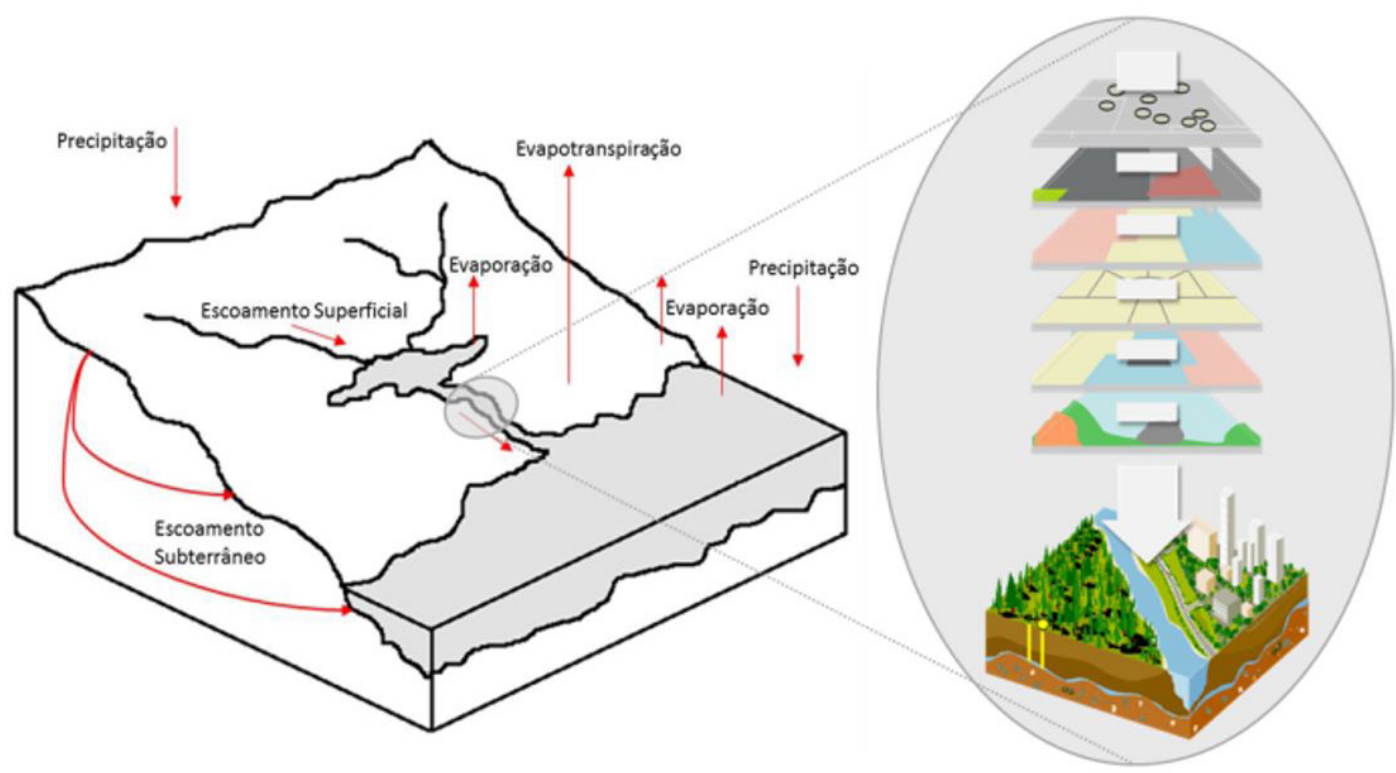

Figura 2 - Sistema representado por um modelo de poluição difusa 
que tange a modelagem da poluição difusa, pois: (1) podem ser locais de difícil representação em modelos hidrológicos em função das dificuldade da modelagem de processos atmosféricos de solo (2) são regiōes de difícil e frequentemente escasso monitoramento (3) são áreas onde frequentemente grandes rios perpassam grandes cidades ou áreas de cultivo agrícola.

A modelagem da poluição difusa é dependente no tipo de ambiente no qual a poluição é gerada, dos processos a serem descritos, dos constituintes do escoamento superficial e de como se processam as suas transformaçôes. Normalmente os processos se dão de maneira diferente em ambientes rurais e urbanos. A maneira como os processos de interceptação, infiltração, e transporte de águas superficiais e subterrâneas, bem como transporte de sedimentos ocorrem sob influência da interferência antrópica e devem ser cuidadosamente reproduzidos. A obtençáo destes dados, especialmente em bacias de médio e grande porte náo é um processo trivial. Modelos de simulação como o SWAT, o AGNPS e o AnnAGNPS, permitem entradas de calendários específicos de cultura, aplicação de fertilizantes e pesticidas ao longo da simulação (YOUNG et al., 1989; BORAH; BERA, 2003; HUANG; HONG, 2010).

Em termos de representação dos processos hidrológicos, os desafios nos ambientes rurais são menores do que aqueles existentes para representar destes em uma bacia urbana. As alteraçóes de uso e ocupação do solo interferem diretamente sobre os processos de evapotranspiração, evaporação, interceptação e, especialmente, infiltração (WANG et al., 2011). Nos ambientes urbanos a dinâmica de recarga do aquífero e separação do escoamento são de difícil representação pelos modelos, a menos que se conheçam todos os pontos de retirada de água e devolução desta ao sistema hídrico, a correta representação destes processos pelos modelos deve considerar as interferências humanas nos processos físicos do ciclo hidrológico (LEANDRO; SCHUMANN; PFISTER., 2016).

Sendo assim, a tarefa de representar processos hidrológicos e de qualidade da água em ambientes de tão elevada heterogeneidade e variabilidade é uma tarefa complexa. Cada processo e suas relações de transferência de massa varia com uma série de fatores, como uso do solo, precipitação, temperatura, vento e evapotranspiração (ERTURK et al., 2007).

\section{SIMULAÇÃO DA POLUIÇÃO DIFUSA}

Os modelos hidrológicos surgiram como ferramentas para melhor entender e representar o comportamento de uma bacia hidrográfica e prever condições diferentes das observadas(SALVADORE; BRONDERS; BATELAAN, 2015). Todo modelo é uma simplificação de determinados processos que ocorrem em um sistema (TUCCI, 1998). Sendo assim, a escolha do melhor modelo para aplicaçáo nos estudos de poluição difusa considerando todos os aspectos associados a esta problemática não é trivial.

O princípio básico na avaliação deste tipo de poluição em bacias hidrográficas, rurais ou urbanas, está na estimativa de carga, uma vez que a carga poluidora lançada varia com a intensidade e duração do evento que o originou, com a extensão da área de produçáo de escoamento naquele evento específico e com outros fatores que inviabilizam a correlação entre carga e vazão (PRODANOFF, 2005; CHEN et al., 2018).

Os modelos de poluição difusa de simulação são o resultado do esforço de produzir códigos que reflitam a integração entre os processos hidrológicos e a qualidade da água como reflexo do uso e ocupação do solo na bacia hidrográfica, possuindo relevantes diferenças estruturais entre si.A grande diferença entra os modelosé a capacidade de representar as características hidrológica, de uso e ocupação do solo e processos de transformação de massana bacia hidrográfica (ZHENG et al., 2014).

Estes modelos possuem graus distintos de complexidade, aplicabilidade e tempo de implementação. Modelos simples são às vezes incapazes de fornecer resultados detalhados, e podem ser ineficientes, e por vezes proibitivas de serem aplicados em bacias maiores ou com certas características (i.e.:tempos de concentração baixos, picos elevados, relaçóes complexas de balanço hídrico e topografia). Nestas aplicaçóes, 
é desejável um balanço entre a simplicidade de modelos mais aproximados, e modelos de computação intensiva. A maioria dos modelos disponíveis hoje foram formuladas nos anos 70, e 80, e desde 1990 têm sido aperfeiçoados ao mesmo tempo que se constroem interfaces gráficas de usuário e integração com sistemas de informação geográfica (BORAH; BERA, 2003).

Por fim, um bom modelo de poluição difusa deve considerar os seguintes fatores: processos climáticos e condiçóes meteorológicas (e.g. Precipitação, temperatura do ar, radiação solar e velocidade do vento), parâmetros de qualidade, incluindo pesticidas, nutrientes, sedimentos de solos superutilizados para pastagens, microrganismos e interaçôes complexas solo-água no fluxo de água e fluxo de solutos considerando atividades naturais a alteraçôes antropogênicas (YANG; WANG, 2010).

\section{REVISÃO DOS MODELOS DE SIM-LAÇÃO DE POLUIÇÃO DIFUSA}

Dentre as diversas possibilidades de modelos a serem utilizados para esta avaliação, a revisão de cada um deles e a definição de critérios para a escolha daquele que melhor se adequa ao problema a ser resolvido ou compreendido é uma etapa importante (SHOEMAKER et al., 2005).

Nesse sentido, Shoemaker et al. (2005) apresenta uma extensa e abrangente revisão acerca dos modelos hidrológicos existentes evidenciando suas principais características em relação aos processos simulados, quantidades de dados de entrada necessários, aspectos de implementação, operacionalidade, e aplicaçóes bem-sucedidas de cada modelo. Neste trabalho, entretanto, maior ênfase foi dada aqueles modelos que atendem os desafios da realidade brasileira, sendo operacionais, ou seja, apresentam, além possuírem de documentação técnica e literatura disponível e possuírem desempenho compatível com a realidade brasileira. Diversos autores destacam (BORAH; BERA, 2003; SALVADORE et al., 2015; HUANG et al., 2010; WANG et al., 2011) como questóes relevantes para a escolha de um modelo: (i) Conhecer os processos físicos mais importantes para a simulação, ou seja, definir qual o tipo de bacia a ser simulada conforme as características de uso do solo, mecanismos e processo de ocupação, natureza do desenvolvimento econômico e social. De maneira geral, podemos distinguir entre as bacias predominantemente rurais ou urbanas, bacias mistas e bacias áridas ou de regime hidrológico convencional.

(ii) Definir qual a possibilidade de obtenção de dados, bem como sua resolução temporal para implementação e calibração.Além disso, avaliar a viabilidade de complementação com técnicas indiretas e medição.

(iii)Avaliar a real necessidade da simulaçáo da poluição difusa a partir dos objetivos de compreensão dos fenômenos e solução de problemas na bacia, levando em conta a complexidade da identificação e quantificação das fontes difusas e pontuais. Este deve levar também em consideração a escala de modelagem (bacia, lote), passos de tempo da simulação e a influência de processos ocorrendo em menor escala, avaliando a compatibilidade da simplificação dos processos coma adequabilidade dos resultados desejados.

(iv) O tempo necessário a implementação do modelo para a obtenção de resultados e tomada de decisão.

A Tabela 1, adaptada de Shoemaker et al. (2005) apresenta um resumo das características de alguns modelos, selecionados de acordo com os critérios acima listados como desejáveis para um modelo dentro da realidade brasileira. A partir do correto estabelecimento das condiçóes de contorno do problema e das informaçóes contidas na tabela, é possível construir uma análise razoável para a escolha de um modelo.

\section{CONCLUSÁO}

As atividades humanas impactama qualidade da água escoada superficialmente. $\mathrm{O}$ mesmo tem sido 


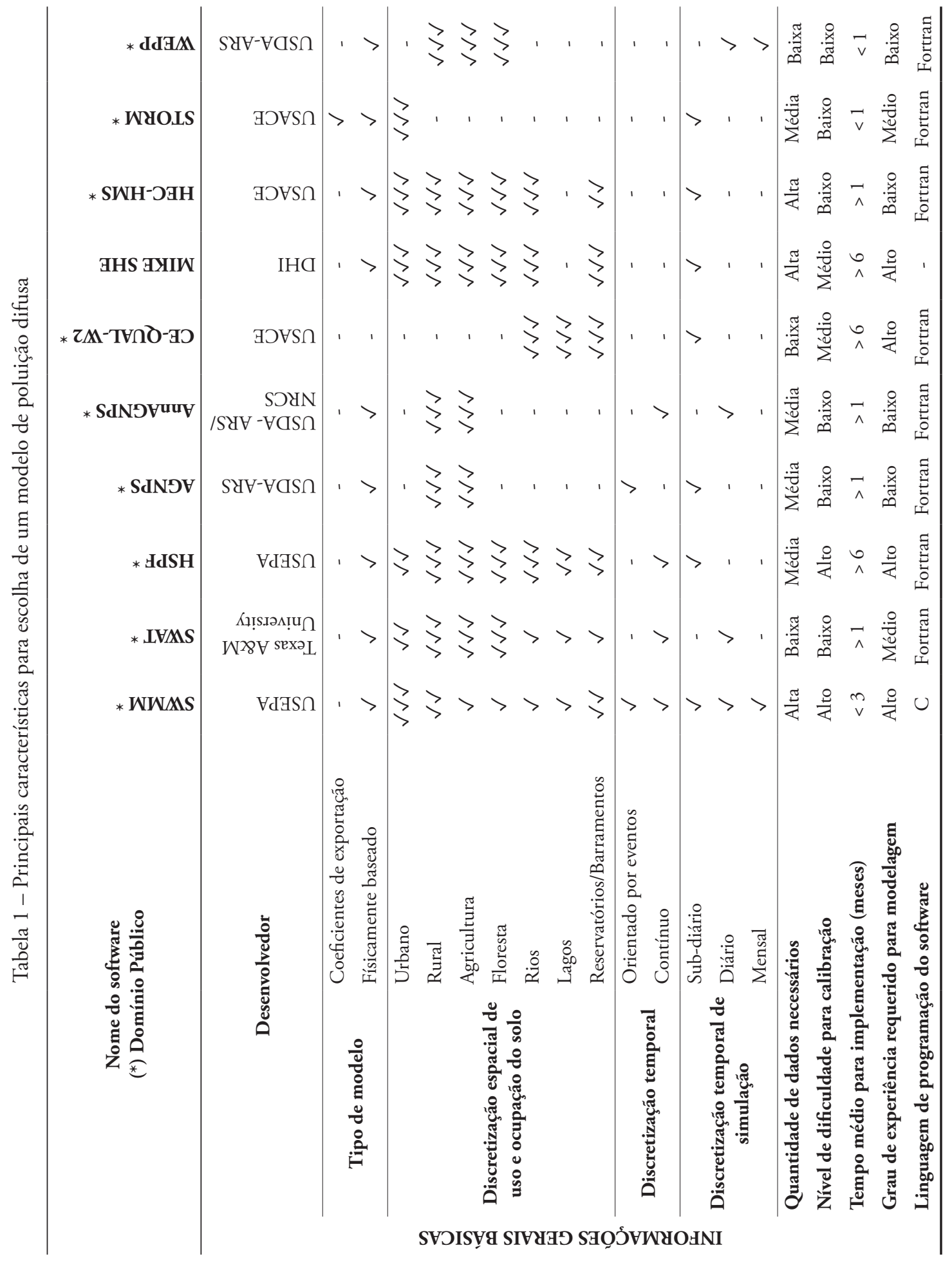




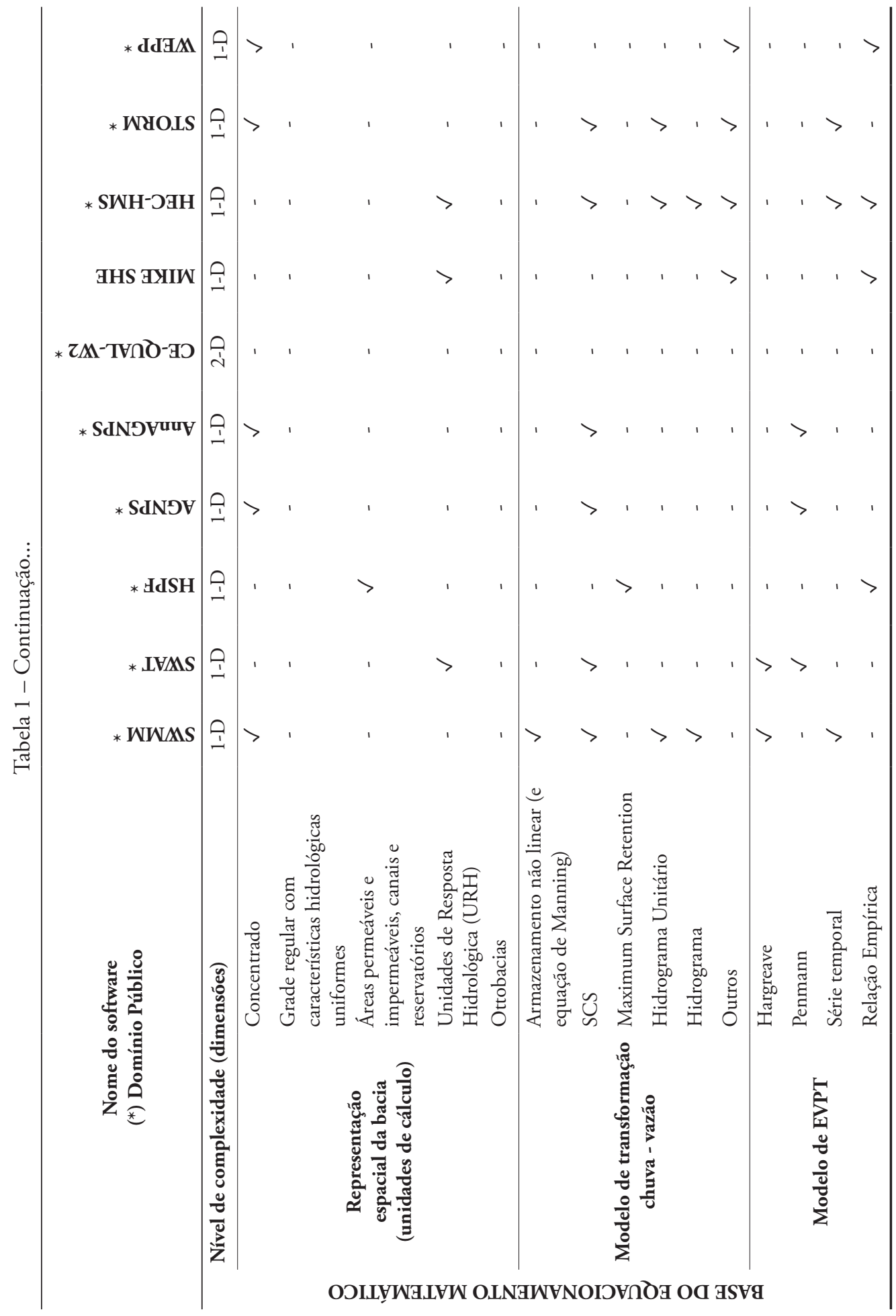




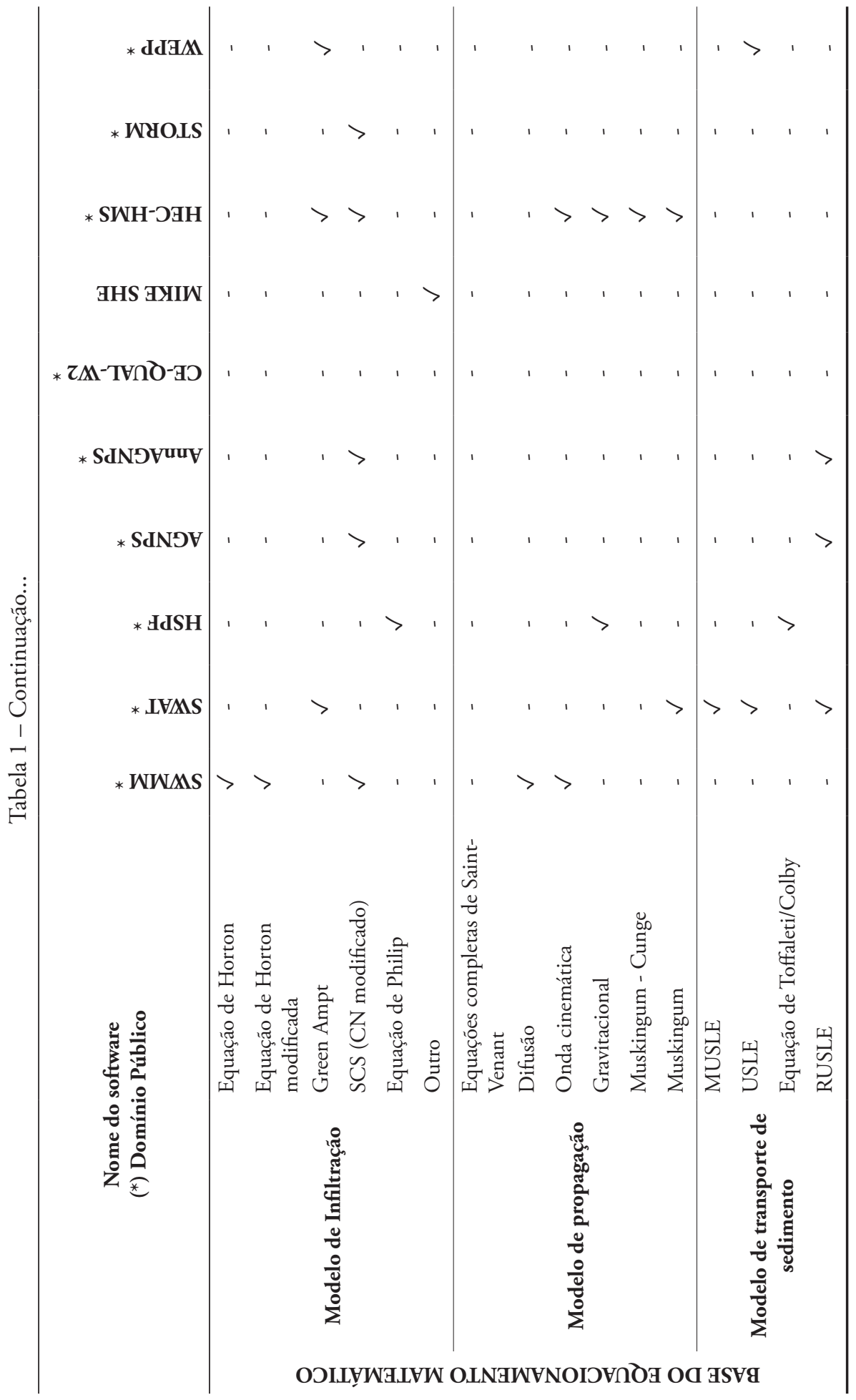




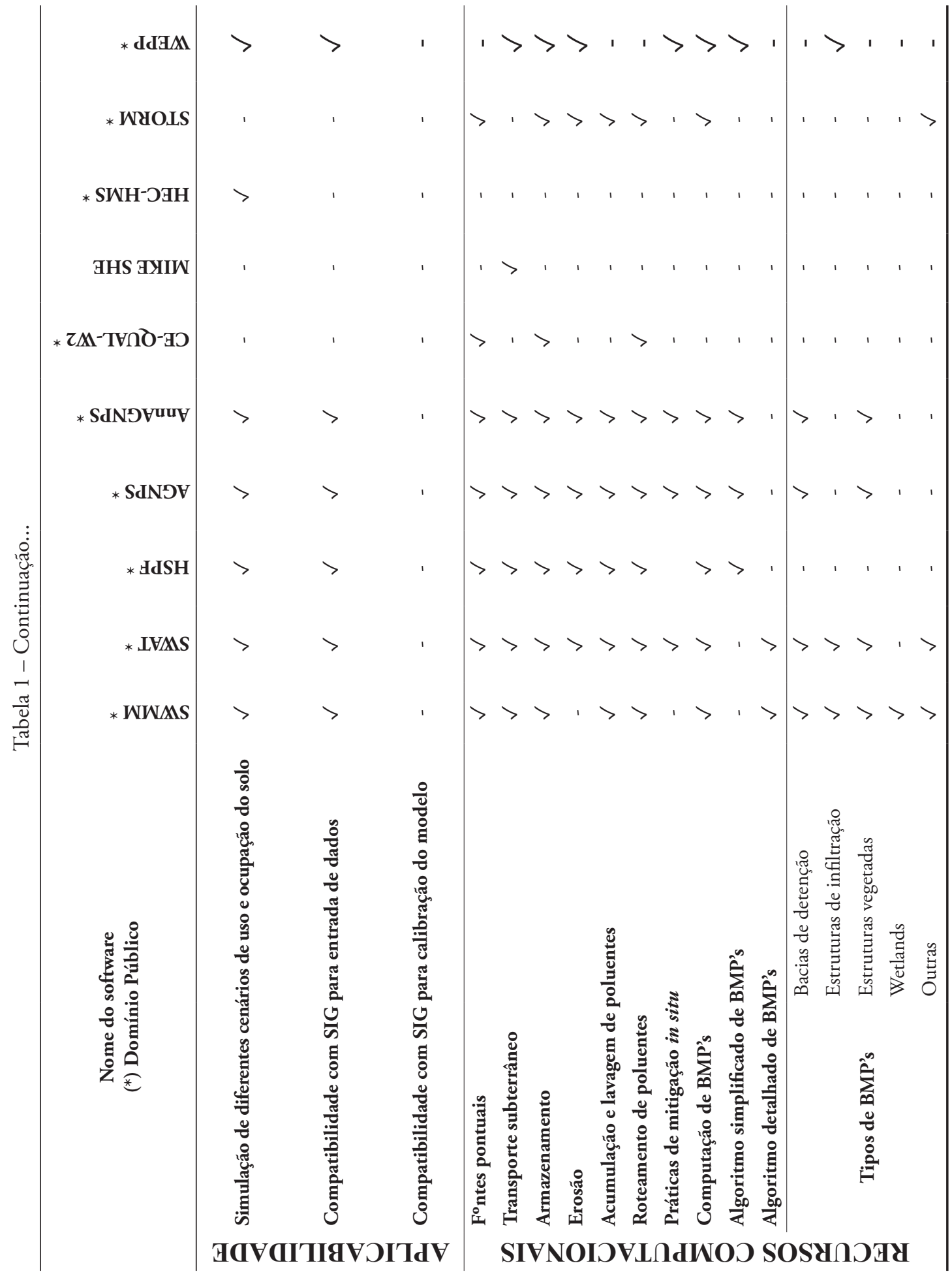




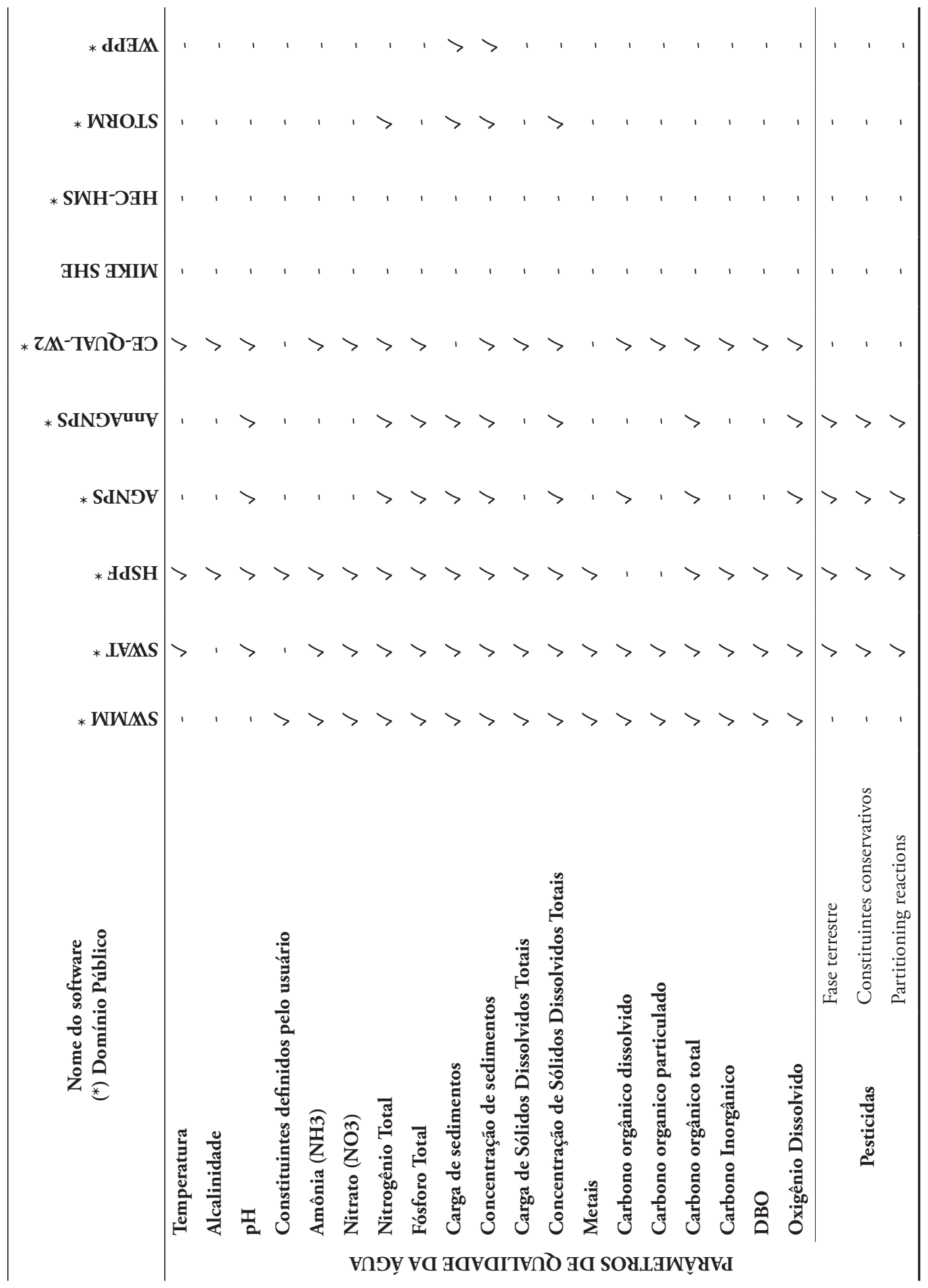


agravado tanto pela impermeabilização das superfícies nos ambientes urbanos, como a crescente utilização de fertilizantes, defensivos e outros insumos industriais e agrícolas. Estas dinâmicas associadas a cenários populacionais e socioeconômicos podem ter impactos severos em populaçóes se constatados problemas de qualidade em suprimentos de abastecimento água.

Aportes excessivos de poluição difusa prejudicam a condição dos corpos aquáticos como ecossistemas, além de causar impedimento de usos para abastecimento, atividades agropecuárias e dessedentação de animais. Em ambientes urbanos, ainda pode trazer ricos à saúde pública, perda da qualidade como objeto recreativo e ativo estético no ambiente urbano.

O sucesso de um programa de controle de poluição difusa, reside basicamente: (i) na coleta sistemática de dados ambientais de quantidade e qualidade, que possam ser associados diretamente aos impactos da poluição difusa e possibilitem entrada em modelos de qualidade da água e/ou indexação de regióes dos modelos de qualidade de água, (ii) na aplicação do processo de tomada de decisão, (iii) na forma como os resultados da modelagem e do monitoramento são apresentados aos decisores e população, de forma a facilitar a comunicação entre estes diferentes atores e atender o objetivo do programa de controle.

O controle da poluição difusa está diretamente associado a identificação de áreas de emissão e estudos de viabilidade de opçôes de mitigação. Técnicas específicas para identificação destas áreas, baseadas em técnicas de análise multicritérios, relaçôes custobenefício ou outras ainda mais abrangentes auxiliam no processo de tomada de decisão. Outra alternativa, nem sempre necessária, a simulação dos processos das bacias tem sido extensivamente utilizada como alternativa, através da modelagem dos processos em ambientes computacionais na escala da bacia hidrográfica. A reprodução destes processos é muito complexa e variável no espaço e tempo.

Estes desafios originam-se desde a coleta de dados de qualidade, nos quais a frequência e o custo do monitoramento podem ser barreiras aos resultados desejados. No entanto, pesquisa recente vem trazendo substitutos à campanhas de amostragem, empregando técnicas indiretas, como derivação de conjuntos de geo-campos e geo-objetos, levantamentos por diferentes e cada vez mais disponíveis fontes de dados, como satélites, aeronaves e drones utilizando tecnologias de alto grau de detalhe, como o Lidar. Embora o emprego de interfaces SIG tenha facilitado o processo de entrada de dados, e aumentado o grau de detalhamento e áreas cada vez maiores, a qualidade e precisão também podem ser limitantes ao modelo que se deseja aplicar ou aos resultados dos mesmos.

Muitos modelos de simulação de poluição foram desenvolvidos desde que os avanços da computação o permitiram. Estes modelos podem ser de complexa e custosa aplicação, além de obrigatoriamente requererem dados de vazão e qualidade da água. Muitos cursos d'água localizam-se em regióes de escassez de dados ou impossibilidade e ou inviabilidade de coleta de dados. Existe uma preocupação com a compatibilidade e aplicabilidade destes modelos, uma vez que são ferramentas poderosas de avaliação de medidas mitigatórias, de convencimento e envolvimento da população como parte integrante da solução do gerenciamento de recursos hídricos.

A escolha de um modelo de simulação passa principalmente pela definição das condiçôes de contorno para o problema a ser resolvido, devendo ser levados em conta aspectos relacionados ao tempo necessário, ao grau de detalhamento esperado e dos dados disponíveis. Os modelos revisados neste artigo atendem a critérios de operacionalidade, de desempenho em regiōes de umidade e balanços hídricos variáveis, podendo ser aplicados em diferentes situaçóes de quantidade de dados, recursos computacionais e processos a serem modelados.

\section{REFERÊNCIAS}

ALMENDINGER, J. E.; ULRICH, J. S. Use of SWAT to estimate spatial scaling of phosphorus export coefficients and load reductions due to agricultural BMPS. Journal of the American Water Resources Association, Herndon, v. 53, n. 3, p. 547-561, 2017.

APRÍGIO, P.; BRANDÃO, J. Non-point source pollution models applied to an urban watershed. In: WORLD ENVIRONMENTAL AND WATER RESOURCES CONGRESS, 2013, Cincinnati, Ohio. Proceedings... Reston: ASCE, 2013. p. 249-265.

BORAH, D. K.; BERA, M. Watershed-scale hydrologic and nonpointsource pollution models: review of mathematical basis. Transactions 
of the ASAE, Michigan, v. 46, p. 1553-1566, 2003. http://dx.doi. org/10.13031/2013.15644.

BRASIL. Ministério do Desenvolvimento Regional. Sistema Nacional de Informações sobre Saneamento Básico - SNSI. Diagnóstico de serviços de água e esgoto do ano de 2015. Brasília: SNSI, 2017. Disponivel em: <http://www.snis.gov.br/diagnostico-agua-e-esgotos/ diagnostico-ae-2015>. Acesso em: 10 ago. 2017.

BURTON, G. A.; PITT, R. E. Stormwater effects handbook: a toolbox for watershed managers scientists, and engineers. Boca Raton: Lewis Publishers, 2001.

CHEN, L. et al. Quantifying nonpoint source emissions and their water quality responses in a complex catchment: a case study of a typical urban-rural mixed catchment. Journal of Hydrology, Amsterdam, v. 559, p. 110-121, 2018.

DAGGUPATI, P. et al. Targeting bmp placement using SWAT sediment yield estimates for fiels-scale BMPs. In: CONFERENCE ON WATERSHED MANAGEMENT TO MEET WATER QUALITY STANDARDS AND EMERGING TMDL, 5., 2010, Baltimore. Proceedings... St. Joseph: ASABE, 2010. p. 14-17.

DIHALA, T. A. et al. Assessing the accuracy of using calibrated HSPF datasets in ungaged watersheds. In: ASAE Conference, 2012, Minessota. Proceedings... Minessota: ASAE, 2012. Paper 002090.

DOBLINSKI, A. F. et al. Nonpoint source pollution by swine farming wastewater in bean crop. Revista Brasileira de Engenharia Agrícola e Ambiental, Campina Grande, v. 14, n. 1, p. 87-93, 2010.

ERTURK,A. et al. Applicability of modelling tools in watershed management for controlling diffuse pollution. Water Science and Technology, London, v. 56, n. 1, p. 147-154, 2007.

ESLAMIAN, S. Handbook of Engineering hydrology: environmental hydrology and water management. In: ESLAMIAN, S. (Org.). Nonpoint source and water quality modelling. Boca Raton: CRC Press, 2014. p. 261-299.

FARZIN, Y. H.; KAPLAN, J. D. Nonpoint source pollution control under Incomplete and Costly information. Environmental and Resource Economics, Boston, v. 28, n. 4, p. 489-506, 2004.

FERRIER, R. C. Diffuse pollution: what is the nature of the problem? Water and Environment Journal, Oxford, v. 19, n. 4, p. 74-366, 2005. http://dx.doi.org/10.1111/j.1747-6593.2005.tb00574.x.

GITAU, M. W.; GBUREK, W. J. L. B. P. Use of the SWAT model to quantify water quality effects of agricultural BMPs at the farmscalelevel. Transactions of the ASABE, St. Joseph, v. 51, n. 2003, p. 1925-1936, 2008.
GOONETILLEKE, A. et al. Understanding the role of land use in urban stormwater quality management. Journal of Environmental Management, London, v. 74, n. 1, p. 31-42, 2005. http://dx.doi.org/10.1016/j. jenvman.2004.08.006.

GURUNG, D. P.; GITHINJI, L. J. M.; ANKUMAH, R. O. Assessing the nitrogen and phosphorus loading in the Alabama (USA) River Basin using PLOAD model. Air, Soil and Water Research, Thousand Oaks, v. 6, p. 23-36, 2012.

HAJKOWICZ, S.; COLLINS, K. A review of multiple criteria analysis for water resource planning and management. Water Resources Management, New York, v. 21, n. 9, p. 1553-1566, 2007.

HARNMEL, R. D. et al. Evaluating, interpreting and communicating performance of hydrologic/water quality models considering intended use: a review and recommendations. Environmental Modelling \& Software, Oxford, v. 57, p. 40-51, 2014.

HUANG, J.; HONG, H. Comparative study of two models to simulate diffuse nitrogen and phosphorus pollution in a medium-sized watershed, southeast China. Estuarine, Coastal and Shelf Science, London, v. 86, n. 3, p. 387-394, 2010.

INSTITUTO BRASILEIRO DE GEOGRAFIAE ESTATÍSTICA - IBGE. Pesquisa nacional de saneamento básico do ano de 2008. Rio de Janeiro: IBGE, 2008. 218 p.

KAINI, P.; ARTITA, K.; NICKLOW, J. W. Evaluating optimal detention pond locations at a watershed scale. World Environmental and Water Resources Congress, Reston, n. 618, p. 1-8, 2007. http://dx.doi. org/10.1061/40927(243)170.

KOO, B. K.; O'CONNELL, P. E. An integrated modelling and multicriteria analysis approach to managing nitrate diffuse pollution: 2. A case study for a chalk catchment in England. The Science of the Total Environment, Amsterdam, v. 358, n. 1-3, p. 1-20, 2006.

KOZAK, C.; FERNANDES, C. V. S. Water quality aassessment and its effects on diffuse pollution considering a new water quality and quantity approach. 2016. Dissertação (Mestrado) - Universidade Federal do Paraná, Curitiba, 2016.

LAABS, V. et al. Fate of pesticides in tropical soils of Brazil under field conditions. Journal of Environmental Quality, Madison, v. 31, n. 1, p. 256, 2002. http://dx.doi.org/10.2134/jeq2002.2560.

LANE, S. N. et al. Surveillant science: challenges for the management of rural environments emerging from the new generation diffuse pollution models. Journal of Agricultural Economics, Reading, v. 57, n. 2, p. 239-257, 2006. 
LEANDRO, J.; SCHUMANN, A.; PFISTER, A. A step towards considering the spatial heterogeneity of urban key features in urban hydrology flood modelling. Journal of Hydrology, Amsterdam, v. 535, p. 356-365, 2016.

LEE, J.; BANG, K. Characterization of urban stormwater runoff. Water Research, New York, v. 34, n. 6, p. 1773-1780, 2000. http://dx.doi. org/10.1016/S0043-1354(99)00325-5.

$\mathrm{LI}$, S. et al. Worldwide performance and trends in nonpoint source pollution modeling research from 1994 to 2013: A review based on bibliometrics. Journal of Soil and Water Conservation, Ankeny, v. 69, n. 4, p. 121A-126A, 2014. http://dx.doi.org/10.2489/jswc.69.4.121A.

LIN, C. et al. Using hydper-spectral indices to detect soil phosphorus concentration for various land use patterns. Environmental Monitoring and Assessment, Dordrecht, v. 187, n. 1, p. 4130-4142, 2015.

LIN, C. et al. Detection of sensitive soil properties related to non-point phosphorus pollution by integrated models of SEDD and PLOAD. Ecological Indicators, New York, v. 60, p. 483-494, 2016.

LIU, S. et al. Fully integrated approach: an alternative solution of coupling a GIS and diffuse pollution models. Frontiers of Environmental Science \& Engineering, Beijing, v. 8, n. 4, p. 616-623, 2014. http:/l dx.doi.org/10.1007/s11783-013-0589-x.

MAILLARD, P.; PINHEIRO SANTOS, N. A. A spatial-statistical approach for modeling the effect of non-point source pollution on different water quality parameters in the Velhas river watershed - Brazil. Journal of Environmental Management, London, v. 86, n. 1, p. 158-170, 2008. http://dx.doi.org/10.1016/j.jenvman.2006.12.009.

MARTIN-MIKLE, C. J. et al. Identifying priority sites for low impact development (LID) in a mixed-use watershed. Landscape and Urban Planning, Amsterdam, v. 140, p. 29-41, 2015. http://dx.doi.org/10.1016/j. landurbplan.2015.04.002.

MILLEDGE, D. G. et al. A Monte Carlo approach to the inverse problem of diffuse pollution risk in agricultural catchments. The Science of the Total Environment, Amsterdam, v. 433, p. 434-449, 2012.

MUNAFÒ, M. et al. River pollution from non-point sources: a new simplified method of assessment. Journal of Environmental Management, London, v. 77, n. 2, p. 93-98, 2005.

NOVOTNY, V. Diffuse pollution and watershed management. 2nd ed. Hoboken: Wiley \& Sons, 2003. p. 50-100.

NOVOTNY, V. Diffuse pollution from agriculture: a worldwide outlook. Water Science and Technology, London, v. 39, n. 3, p. 1-13, 1999. http://dx.doi.org/10.2166/wst.1999.0124.
OMERNIK, J. M. EPA-600/3-76-104: the influence on land use on stream nutrient levels. Corvallis: United States Environmental Protection Agency, 1976.

OZCAN, Z. et al. A pollution fate and transport model in a semi-arid region: is some number better than no number? The Science of the Total Environment, Amsterdam, v. 595, p. 425-440, 2017a.

ÖZCAN, Z.; KENTEL, E.; ALP, E. Evaluation of the best management practices in a semi-arid region with high agricultural activity. Agricultural Water Management, Amsterdam, v. 194, p. 160-171, 2017b. http:// dx.doi.org/10.1016/j.agwat.2017.09.007.

PANAGOPOULOS, Y. et al. SWAT parametrization for the identification of critical diffuse pollution sources areas under data limitations. Ecological Modelling, Amsterdam, v. 222, n. 19, p. 3500-3512, 2011. http://dx.doi. org/10.1016/j.ecolmodel.2011.08.008.

PARKINSON, J.; MARK, O. Urban stormwater management in developing countries. Estados Unidos: IWA, 2005. p. 10.

PETERSEN, G. W. et al. Evaluation of agricultural nonpoint pollution potential in Pennsylvania using a geographic information system. Harrisburg, 1991.

PRODANOFF, J. H. A. Avaliação da poluição difusa gerada por enxurradas em meio urbano. 2005. 266 f. Tese (Doutorado em Engenharia Civil) - Universidade Federal do Rio de Janeiro, Rio de Janeiro, 2005.

RIGHETTO, A. M.; GOMES, K. M.; FREITAS, F. R. S. Poluição difusa nas águas pluviais de uma bacia de drenagem urbana. Engenharia Sanitária e Ambiental, Rio de Janeiro, v. 22, n. 6, p. 1109-1120, 2017.

ROMAGNOLI, M. et al. Assessment of of the Swat model to simulate a watershed with limited data available in the Pampas region, Argentina. The Science of the Total Environment, Amsterdam, v. 596-597, p. 437-450, 2017.

SALVADORE, E.; BRONDERS, J.; BATELAAN, O. Hydrological modelling of urbanized catchments: a review and future directions. Journal of Hydrology, Amsterdam, v. 529, p. 62-81, 2015.

SHAVER, E. et al. Fundamentals of urban runoffmanagement: technical and institutional issues. Washington: United States. Environmental Protection Agency, 1994. Disponível em: <http://agris.fao.org/agrissearch/search/display.do?f=1995/US/US95046.xml;US9536404>. Acesso em: 15 ago. 2017.

SHEN, Z. et al. A framework for priority non-point source area identification and load estimation integrated with APPI and PLOAD model in Fujiang Watershed, China. Agricultural Water Management, Amsterdam, v. 98, n. 6, p. 977-989, 2011. 
SHEN, Z. Y. et al. A comprehensive study of the effect of GIS data on hydrology and non-point source pollution modelling. Agricultural Water Management, Amsterdam, v. 118, p. 93-102, 2013. http://dx.doi. org/10.1016/j.agwat.2012.12.005.

SHI, Q. et al. Best management practices for agricultural non-point source pollution control using PLOAD in Wuliangsuhai watershed. Journal of Food Agriculture and Environment, Helsinki, v. 10, n. 2, p. 1389-1393, 2012.

SHOEMAKER, L. et al. TMDL model evaluation and research needs. EPA, 2005. 403 p.

TAN, M. L. et al. Impacts of DEM resolution, source, and resampling technique on SWAT-simulated streamflow. Applied Geography, Sevenoaks, v. 63, p. 357-368, 2015.

TUCCI, C. E. M. Modelos hidrológicos. 1. ed. Porto Alegre:ABRH, 1998.

UNITED STATES ENVIRONMENTAL PROTECTIONAGENCY - USEPA. Hydrological simulation program (FORTRAN): user's manual for release 10. Washington: USEPA, 1993.

UNITED STATES ENVIRONMENTAL PROTECTIONAGENCY - USEPA. Preliminary data summary of urban stormwater BMP's. Washington: USEPA, 1999.
WANG, L. et al. Urban nonpoint source pollution buildup and wash-off models for simulating storm runoff quality in the Los Angeles County. Environmental Pollution, Barking, v. 159, n. 7, p. 1932-1940, 2011. http://dx.doi.org/10.1016/j.envpol.2011.03.019.

WARDROPPER, C. B.; GILLON, S.; RISSMAN, A. R. Uncertain monitoring and modelling in a watershed nonpoint pollution program. Land Use Policy, Guildford, v. 67, p. 690-701, 2017.

WILLIS, W. B. et al. Lidar method to estimate emission rates from extended sources. Journal of Atmospheric and Oceanic Technology, Boston, v. 34, n. 2, p. 335-345, 2016.

YANG, P. et al. What is the effect of LiDAR-derived DEM resolution on large-scale watershed model results? Environmental Modelling \& Software, New York, v. 58, p. 48-57, 2014.

YANG, Y. S.; WANG, L. A review of modelling tools for implementation of the EU Water framework directive in handling diffuse water pollution. Water Resources Management, New York, v. 24, n. 9, p. 1819-1843, 2010. http://dx.doi.org/10.1007/s11269-009-9526-y.

YOUNG, R. A. et al. AGNPS: a nonpoint-source pollution model for evaluating agricultural watersheds. Journal of Soil and Water Conservation, Ankeny, v. 44, n. 2, p. 168-173, 1989.

ZHENG, Y. et al. Addressing the uncertainty in modeling watershed nonpoint source pollution. Developments in Environmental Modelling, New York, v. 26, p. 113-159, 2014.

Contribuição do autor:

Revisão Bibliográfica, discussão dos resultados, revisão do texto.

Ana Paula Mühlenhoff

Universidade Federal do Paraná. anapaulamuhlenhoff@gmail.com

Contribuição do autor:

Revisão Bibliográfica, discussão dos resultados.

Cristóvão Vicente Scapulatempo Fernandes

Universidade Federal do Paraná. cris.dhs@ufpr.br

Contribuição do autor: 